Article

\title{
A Comparative Theoretical Study of Picric Acid and Its Cocrystals
}

\author{
Peng-Yuan Chen, Lin Zhang *, Shun-Guan Zhu and Guang-Bin Cheng \\ School of Chemical Engineering, Nanjing University of Science and Technology, Nanjing 210094, \\ China; E-Mails: chenpengyuan@outlook.com (P.-Y.C.); zhusg@mail.njust.edu.cn (S.-G.Z.); \\ gcheng@mail.njust.edu.cn (G.-B.C.) \\ * Author to whom correspondence should be addressed; E-Mail: zhangl@mail.njust.edu.cn; \\ Tel./Fax:+86-25-8431-5856.
}

Academic Editor: Thomas M. Klapötke

Received: 15 July 2015 / Accepted: 20 August 2015 /Published: 1 September 2015

\begin{abstract}
A novel cocrystal of picric acid/acetophenone was prepared by solvent evaporation method and the crystal structure was characterized by single crystal X-ray diffraction. Analysis of the crystal structure shows that the hydrogen bonding, van der Waals and $\pi-\pi$ stacking are the main driving forces for the cocrystal formation. Density functional theory (DFT) calculation was performed to better understand the formation mechanism and properties of the cocrystal. The results suggest that $\pi-\pi$ stacking is more important than hydrogen bonding considering their interaction energies. Furthermore, Mulliken charge analysis shows picric acid becomes less sensitive after cocrystallization with other compounds because of the electron transfer.
\end{abstract}

Keywords: picric acid; cocrystal; DFT; hydrogen bonding; $\pi-\pi$ stacking

\section{Introduction}

Insensitive high energy density materials (IHEDMs) have attracted considerable attention in recent years because of their insensitivity to external stimuli, good detonation performance and high thermal stability [1]. However, there is always a conflict between the detonation performance and sensitivity. High detonation performance usually goes with high sensitivity [2,3]. As a result, few explosives can meet the rigorous requirements. Although a lot of new explosives were synthesized and many existing 
explosives were modified by different methods, little progress has been achieved [4,5]. Recently, cocrystallization, the formation of a crystal with two or more neutral species in a definite ratio with distinct solid-state properties, is emerging as a promising way to alter the properties of energetic materials, such as melting point, sensitivity, detonation performance, and so on [6-8].

Seventeen cocrystals of TNT (2,4,6-trinitrotoluene) were reported by Landenberger et al.[9]. The donor-acceptor $\pi-\pi$ interaction is the driving force for the cocrystal formation. The sensitivity of the cocrystal reduces a lot but the energy is diluted too because of the intervention of non-energetic materials. Bolton et al. got the cocrystal of TNT/CL-20 (2,4,6,8,10,12-hexanitrohexaazaisowurtzitane) by solvent evaporation method [10]. This cocrystal forms based on a series of $\mathrm{CH}$ hydrogen bonds between nitro group oxygens and aliphatic hydrogens as well as interactions between the electron-deficient ring of TNT and nitro groups of CL-20. The cocrystal has a lower sensitivity compared with the pure CL-20. The cocrystal of HMX(1,3,5,7-tetranitro-1,3,5,7-tetrazocane)/CL-20 was predicted to be more powerful than HMX. Nevertheless, the sensitivity of the cocrystal is no higher than that of HMX [11].

Due to the strong acidity, picric acid is easy to form salts with metal. Its lead salt is widely used as primary explosive. In addition, picric acid is likely to form cocrystals with other substances, especially aromatic compounds [12-14]. In this paper, an unreported cocrystal of picric acid was synthesized and characterized by single crystal X-ray diffraction. Initially, $\pi$ - $\pi$ stacking was considered as the main driving force for the cocrystal formation. With the developing of research in energetic cocrystals, more driving forces were recognized, including weak hydrogen bond and electrostatic interaction. In many cases, more than one kind of intermolecular interaction appears in the cocrystal, and a comprehensive understanding of each interaction and their magnitude is helpful in cocrystal designing. Therefore, density functional theory calculations were performed based on the crystal structures $[15,16]$. Besides the cocrystal of picric acid/acetophenone, picric acid and two other cocrystals of picric acid were also calculated for comparison. Although acetophenone, 2-nitroaniline and 4-nitroaniline have different functional groups, all the three cocrystals are constructed by $\pi-\pi$ stacking and weak hydrogen bond. The cocrystals of picric acid/acetophenone, picric acid/2-nitroaniline[17] and picric acid/4-nitroaniline [18] are represented by cocrystal 1 , cocrystal 2 and cocrystal 3 for brevity.

\section{Experimental Section}

\subsection{Synthesis}

A total of $1 \mathrm{~g}$ picric acid was added to $25 \mathrm{~mL}$ acetophenone with stirring for $30 \mathrm{~min}$. The solution was filtered and then the filtrate was evaporated at room temperature until colorless crystal appeared.

\subsection{X-ray Crystallography}

The single-crystal X-ray diffraction data of the cocrystal were collected on Bruker SMART-APEX CDD diffraetometer (Bruker AXS Inc., Madison, WI, USA) by using Mo K $\alpha$ X-ray radiation (Bruker AXS Inc., Madison, WI, USA) $(\lambda=0.71069 \AA)$ and a graphite monochromator.

Powder X-ray diffraction (PXRD) pattern for the cocrystal was taken from a Bruker D8 Advance (Bruker AXS Inc., Madison, WI, USA) (Cu K $\alpha$ radiation, voltage $40 \mathrm{kV}$, and current $40 \mathrm{~mA}$ ). The data were collected over an angle ranging from $5^{\circ}$ to $40^{\circ}$ with a scanning speed of $0.02^{\circ}$ per second. 


\subsection{Computational Method}

The initial structures were taken from X-ray diffraction. MPW1B95 density functional and 6-311++G $(2 \mathrm{~d}, 2 \mathrm{p})$ basis set were employed to full optimize the geometries of all the complexes. Binding energies and Mulliken charges were calculated based on the optimized structures. All the calculations were performed in Gaussian 09.

\section{Results and Discussion}

\subsection{Crystal Structure}

The crystal structure was solved by the direct method and refined by the full-matrix least-squares on $\mathrm{F}^{2}$ by using SHELXTL-97 program [19]. The crystal data has been deposited to the Cambridge Crystallographic Data Center, the CCDC number is 965984 . The crystal data of picric acid/acetophenone cocrystal is presented in Table 1 . The cocrystal is formed by picric acid and acetophenone molecules in a ratio of $1: 1$.

Table 1. The crystal data of picric acid/acetophenone cocrystal.

\begin{tabular}{lc}
\hline Parameter & Data \\
\hline Empirical formula & $\mathrm{C}_{6} \mathrm{H}_{3} \mathrm{~N}_{3} \mathrm{O}_{7} \cdot \mathrm{C}_{7} \mathrm{H}_{8} \mathrm{O}$ \\
\hline Formula weight & $349.26 \mathrm{~g} / \mathrm{mol}$ \\
\hline Temperature & $293(2) \mathrm{K}$ \\
\hline Crystal system & Monoclinic \\
\hline Space group & $\mathrm{P} 21 / \mathrm{c}$ \\
\hline & $a=8.494(18) \AA$ \\
Cell parameters & $b=17.41(4) \AA$ \\
& $c=10.18(2) \AA$ \\
\hline Volume & $\beta=92.76^{\circ}(4)$ \\
\hline Z & $1504(5) \AA^{3}$ \\
\hline Calculated density & 4 \\
\hline Absorption coefficient & $1.543 \mathrm{~g} / \mathrm{cm}^{3}$ \\
\hline F(000) & $0.129 \mathrm{~mm}{ }^{-1}$ \\
\hline Crystal size & 720 \\
\hline Theta range for data collection & $2.32-28.25^{\circ}$ \\
\hline Max. and min. transmission & $0.9879 \mathrm{and} 0.9872$ \\
\hline Data/restraints/parameters & $3427 / 0 / 226$ \\
\hline Goodness-of-fit on $\mathrm{F}^{2}$ & 1.005 \\
\hline Final R indices [I $>2$ sigma(I)] & $\mathrm{R} 1=0.0754, \mathrm{wR} 2=0.1615$ \\
\hline R indices (all data) & $\mathrm{R} 1=0.1652, \mathrm{wR} 2=0.1993$ \\
\hline
\end{tabular}

As is depicted in Figure 1, the acetophenone molecule has a weak interaction with the adjacent picric acid molecules. 


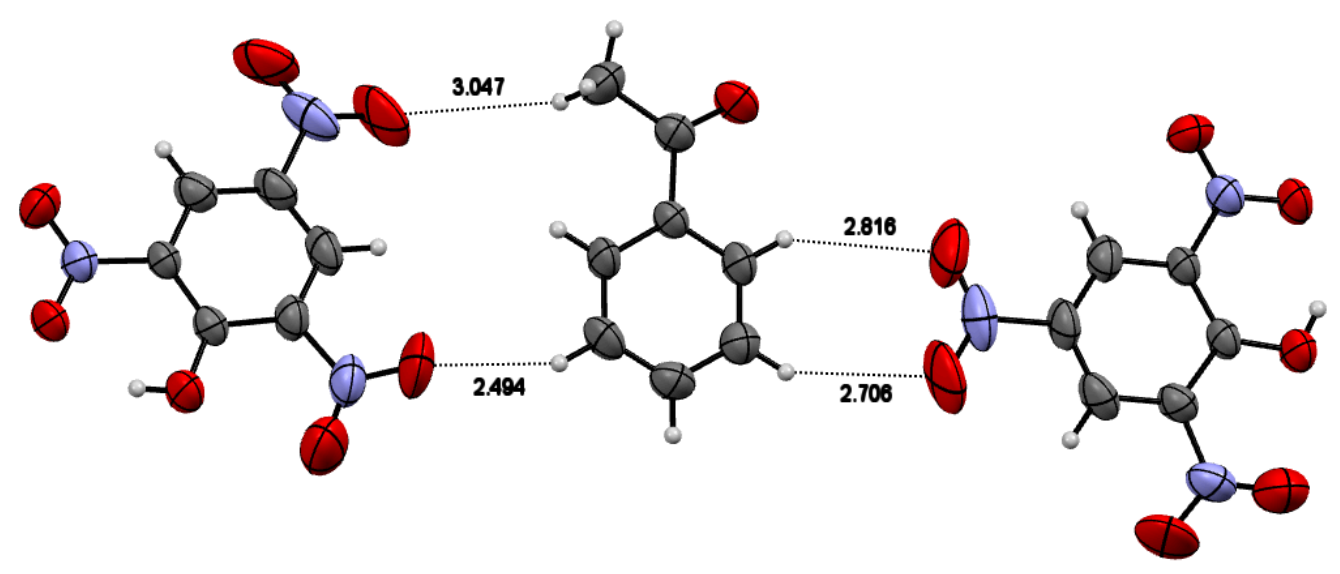

(a)

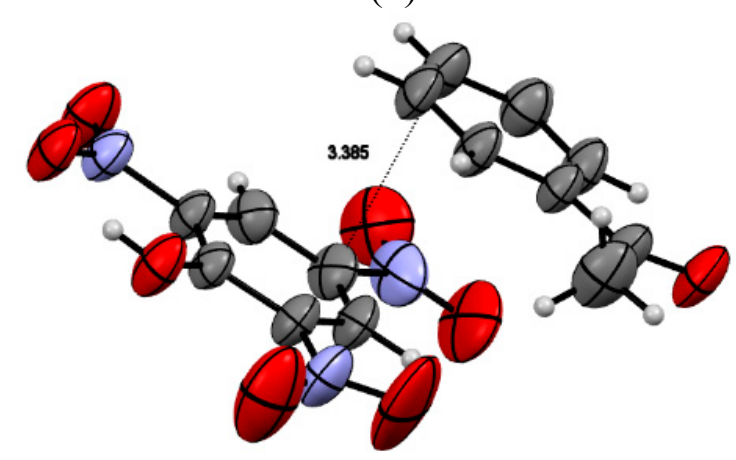

(b)

Figure 1. Intermolecular interactions between picric acid and acetophenone in the cocrystal:

(a) hydrogen bonding; (b) $\pi$ - $\pi$ stacking. Displacement ellipsoids are drawn at the $50 \%$ level.

The nitro group of picric acid forms $\mathrm{C}-\mathrm{H}$... $\mathrm{O}-\mathrm{NO}$ weak hydrogen bond with the $\mathrm{H}$ in the benzene ring of acetophenone, and the bond lengths are $2.494 \AA, 2.706 \AA$ and $2.816 \AA$, respectively (The corresponding bond angles are $152^{\circ}, 150^{\circ}$, and $144^{\circ}$, respectively). Furthermore, the $\mathrm{H}$ of methyl in acetophenone forms a weaker $\mathrm{C}-\mathrm{H}$... $\mathrm{O}-\mathrm{NO}$ intermolecular hydrogen bond with the nitro group of picric acid, with a bond length of $3.047 \AA$ and bond angle of $136^{\circ}$. These intermolecular interactions are not very strong but make a lot of contributions in stabilizing the system. The "face to face" molecules, picric acid and acetophenone, is almost parallel with a dihedral angle of $2.94^{\circ}$. The shortest distance of $\mathrm{C}-\mathrm{C}$ in respective benzene ring is $3.385 \AA$. Usually, when the vertical distance between two rings is in the region of 3.3-3.8 $\AA$, the $\pi-\pi$ stacking formed [20]. Since the vertical distance between the two rings in the cocrystal is between 3.28-3.44 $\AA, \pi-\pi$ stacking can be regarded as another driving force for the crystal formation.

From Figure 2, viewed along the a-axis, "face to face" $\pi$ - $\pi$ stacking is observed in the cocrystal. While viewed along the b-axis, the molecules form an interleaved layered structure. No notable hydrogen bonding was found, Van der Waals force is the main intermolecular interaction. The layered structure like FOX-7 (1,1-diamino-2,2-dinitroethene) and TATB (1,3,5-triamino-2,4,6-trinitrobenzene) is helpful to dilute the external force [21]. Therefore, it can be deduced that the cocrystal is much more insensitive than pure picric acid. 


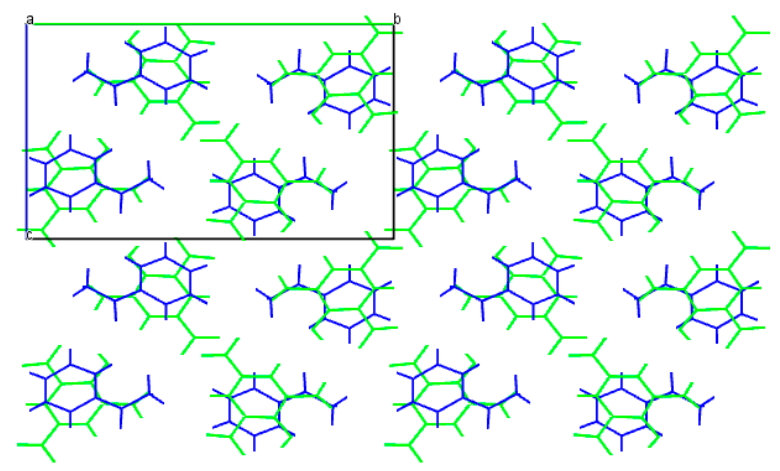

(a)

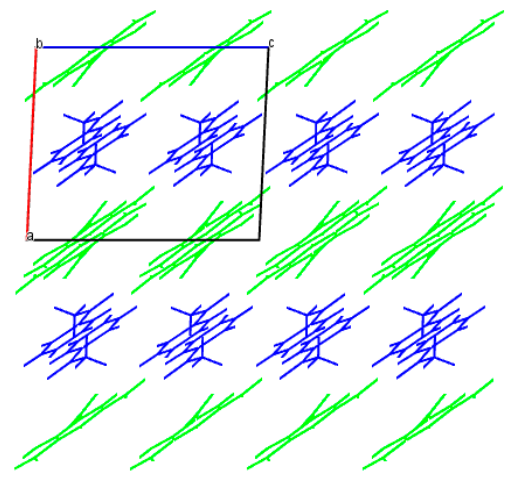

(b)

Figure 2. Cocrystal packing diagram viewed along the a-axis (a) and b-axis (b).

\subsection{PXRD Analysis}

PXRD is widely used for the detection of polymorphism and it is a useful tool to judge the phase purity of bulk samples with single crystals. From Figure 3, it is obvious that PXRD pattern of the bulk sample is almost the same with that simulated form crystal structure, indicating the bulk sample is cocrystal 1 with a high phase purity. The differences in relative diffraction intensity for the same crystallographic index between the two patterns are considered to be caused by the preferred orientation of the bulk samples.

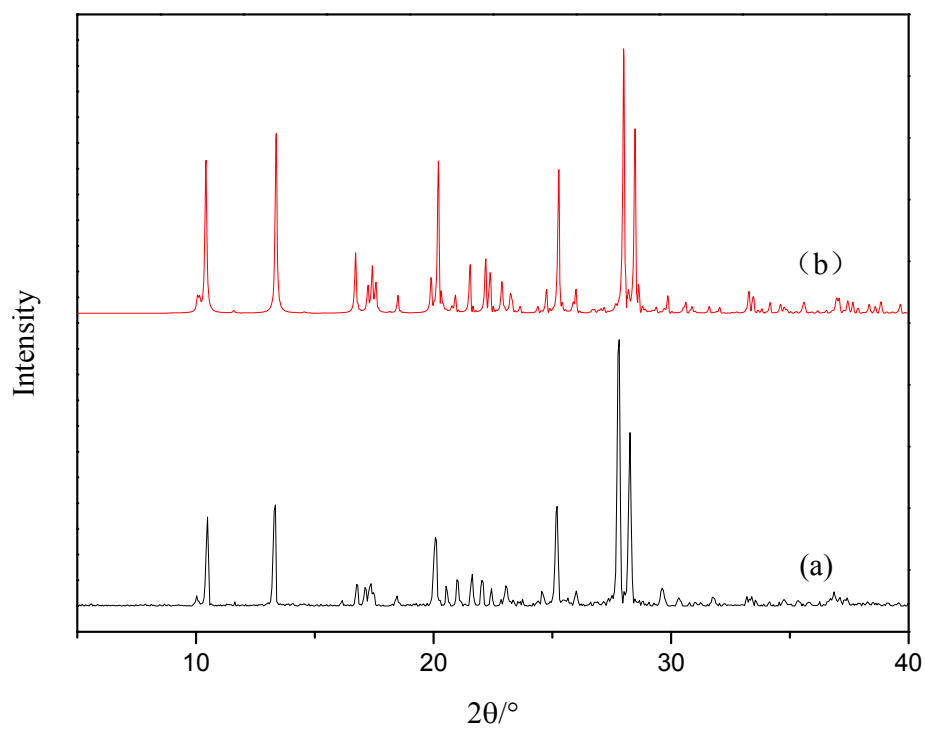

Figure 3. PXRD patterns of (a) cocrystal 1 and that (b) simulated from crystal structure.

\subsection{Hirshfeld Surface Analysis}

In order to further investigate the influence of different coformer on the intermolecular interactions experienced by picric acid, CrystalExplorer was employed to calculate the various contact contributions to Hirshfeld surface area in four crystals [22]. From Table 2, we can see that the percentage of the O...H interaction in three cocrystals is obviously higher than that in picric acid. It illustrates that hydrogen bonding and Van der Waals force play an important role in cocrystal formation. Moreover, there is no 
C...C interaction in picric acid, while it exists in three cocrystals. That is to say, $\pi$ - $\pi$ stacking exists in three cocrystals [23]. Then we can deduce that the $\pi-\pi$ stacking is another factor that affects the formation of the cocrystals. This conclusion is consistent with the crystal structure analysis of cocrystal 1.

Table 2. Various contact contributions to Hirshfeld surface area in four crystals.

\begin{tabular}{ccccc}
\hline Interaction & Picric Acid & Cocrystal 1 & Cocrystal 2 & Cocrystal 3 \\
\hline O...O & 26.5 & 13.9 & 14.5 & 21.9 \\
O...H & 33.7 & 49.6 & 54.9 & 41.1 \\
O... & 26.1 & 11.1 & 1.6 & 16.0 \\
O... & 10.7 & 3.9 & 4.0 & 8.8 \\
H...H & 0.8 & 4.4 & 7.0 & 2.4 \\
H... & 0.2 & 7.5 & 2.7 & 4.3 \\
C...C & 0 & 3.9 & 10.0 & 2.9 \\
\hline
\end{tabular}

\subsection{Binding Energy and Mulliken Charge}

From Figure $4 \mathrm{a}$, it is obvious that the binding energy of $\pi-\pi$ stacking is almost triple that of a hydrogen bonding. That is to say, $\pi-\pi$ stacking plays a dominant role in the formation of cocrystal 1 . The same phenomenon appears in cocrystal 2 and cocrystal 3 . In cocrystal 2 , the binding energies of $\pi$ - $\pi$ stacking between picric acid and 2-nitroaniline are almost equal and are larger than that between two 2-nitroaniline molecules. Additionally, the hydrogen bonding energy between two 2-nitroaniline molecules is larger than that between 2-nitroaniline and picric acid. Picric acid has a more homogeneous electron distribution at the edge of the molecule compared with 2-nitroaniline. As a result, the electrostatic attraction between two 2-nitroaniline molecules is larger than that between picric acid and 2-nitroaniline in the hydrogen bonding complex.

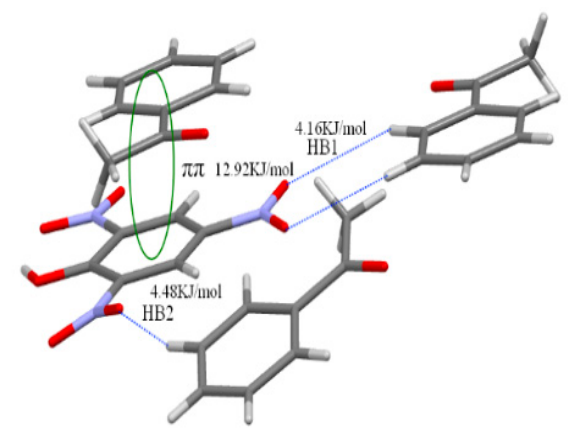

(a)

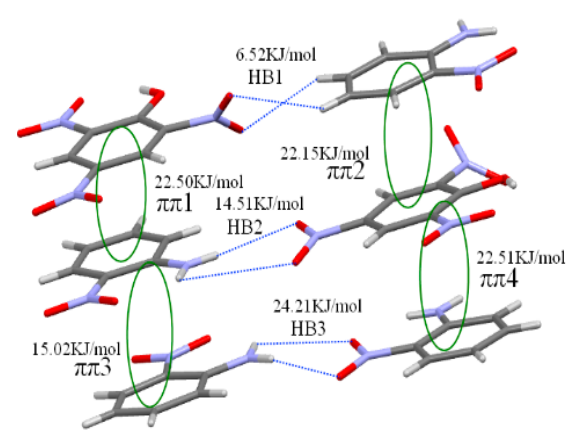

(b)

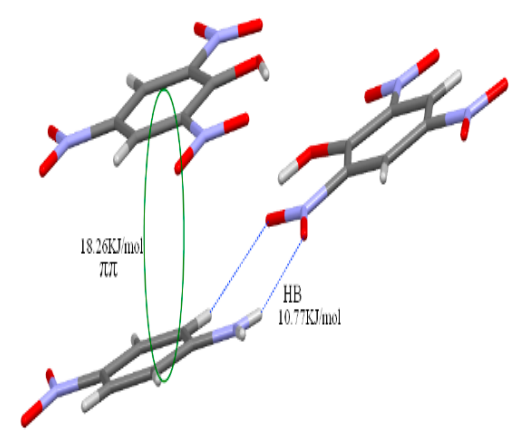

(c)

Figure 4. The energetic contributions of intermolecular $\pi-\pi$ interactions and hydrogen bonding interactions in: (a) cocrystal 1; (b) cocrystal 2; (c) cocrystal 3.

Based on the analysis of crystal structure and Hirshfeld surface, we can deduce that the main driving forces of the cocrystals formation are weak hydrogen bonding and $\pi$ - $\pi$ stacking interaction. Nevertheless, the quantitative contribution of each force is not clear. Since Mulliken charge has a close relationship with the properties of energetic materials, MPW1B95 density functional and 6-311++G (2d, 2p) basis set were employed to calculate the binding energies of $\pi$ - $\pi$ stacking and weak hydrogen bonding as well 
as Mulliken charges. It was found that counterpoise correction is not necessary when this functional is used with a triple- $\zeta$ quality or larger basis set [24].

Mulliken charges of some selected atoms, like $\mathrm{O}, \mathrm{N}, \mathrm{H}$, which forms the hydrogen bonds in picric acid and cocrystals are listed in Table 3.

Table 3. The calculated Mulliken charges of picric acid and three cocrystals.

\begin{tabular}{|c|c|c|c|c|c|c|c|}
\hline Crystal & $\begin{array}{c}\text { Interac } \\
\text { tion }\end{array}$ & Molecule & 01 & $\mathbf{O 2}$ & $\mathbf{N}$ & H1 & H2 \\
\hline \multirow{4}{*}{ Cocrystal1 } & \multirow{2}{*}{ HB1 } & picric acid & -0.098 & -0.093 & 0.019 & 0.185 & 0.167 \\
\hline & & complex & -0.003 & -0.004 & -0.174 & 0.183 & 0.170 \\
\hline & \multirow{2}{*}{ HB2 } & picric acid & -0.093 & -0.092 & - & 0.139 & 0.169 \\
\hline & & complex & -0.066 & -0.069 & - & 0.162 & 0.221 \\
\hline \multirow{6}{*}{ Cocrystal2 } & \multirow{2}{*}{ HB1 } & picric acid & -0.092 & -0.050 & -0.052 & 0.169 & 0.168 \\
\hline & & complex & 0.006 & 0.039 & -0.248 & 0.178 & 0.128 \\
\hline & \multirow{2}{*}{ HB2 } & picric acid & -0.098 & -0.093 & 0.019 & 0.178 & 0.136 \\
\hline & & complex & -0.022 & -0.019 & -0.159 & 0.258 & 0.157 \\
\hline & \multirow{2}{*}{ HB3 } & picric acid & -0.148 & -0.194 & 0.059 & 0.136 & 0.178 \\
\hline & & complex & -0.080 & -0.066 & -0.154 & 0.185 & 0.272 \\
\hline \multirow{2}{*}{ Cocrystal3 } & \multirow{2}{*}{ HB1 } & picric acid & -0.080 & -0.058 & -0.015 & 0.179 & 0.143 \\
\hline & & complex & -0.033 & 0.023 & -0.255 & 0.244 & 0.129 \\
\hline
\end{tabular}

The charge redistribution mainly occurs on the adjacent NO...H atoms which form hydrogen bond. Compared with coformer, the entire oxygen atom in the nitro group in the cocrystals has fewer electrons. However, the nitrogen atom in nitro group acquires electrons when the cocrystal comes into being. The Mulliken charges of the hydrogen atoms do not show a big change. As a whole, the net charge of picric acid all increase and it is positively charged with electricity, indicating electrons transfer from acetophenone, 2-nitroaniline, and 4-nitroaniline to picric acid. It is notable that, in cocrystal 2, the net charge changes in different complexes are $0.0067,0.0170$ and 0.2356 e, respectively, while the binding energies for them are $6.52,14.51$ and $24.21 \mathrm{~kJ} / \mathrm{mol}$, they show the same order. Hence, net charge transfer has a relationship with binding energy and maybe can be used as a criterion to judge the stability of the complex.

The calculated total Mulliken charge of nitro group in picric acid molecule is -0.609 e, while it becomes -0.688 e in cocrystal $1,-0.81$ e, -0.673 e, -0.81 e(different complex) in cocrystal 2 , and -0.684 e in cocrystal 3 , respectively. The more negative charges the nitro group possesses, the more stable the nitro compound becomes [25]. Then it can be concluded that the sensitivity of three cocrystals is lower than picric acid, which is consistent with the analysis of the crystal structure.

\section{Conclusions}

In order to assist in a better understanding of the formation mechanism of cocrystal explosive, a new cocrystal was synthesized and characterized. DFT calculation was also performed based on the experimental crystal structure. The cocrystal of picric acid/acetophenone was obtained by solvent evaporation method. The analysis of the crystal structure and Hirshfeld surface indicates that the hydrogen bonding and $\pi-\pi$ stacking interactions are the main driving force for the cocrystal formation. 
Additionally, the binding energy of the $\pi-\pi$ stacking is larger than the hydrogen bonding, suggesting the former plays a dominant role in the cocrystal formation. The total Mulliken charge on the nitro group of the picric acid molecule in cocrystal is larger than that in pure picric acid, indicating the cocrystals are less sensitive than picric acid.

\section{Author Contributions}

Lin Zhang conceived the project, Peng-Yuan Chen performed the experiments and analyzed the data under the direction of Lin Zhang, Shun-Guan Zhu, and Guang-Bin Cheng. All authors contributed in the manuscript preparation.

\section{Conflicts of Interest}

The authors declare no conflict of interest.

\section{References}

1. Lin, H.; Zhu, S.G.; Li, H.Z.; Peng, X.H. Synthesis, characterization, AIM and NBO analysis of HMX/DMI cocrystal explosive. J. Mol. Struct. 2013, 1048, 339-348.

2. Li, S.H.; Wang, Y.; Qi, C.; Zhao, X.X.; Zhang, J.C.; Zhang, S.W.; Pang, S.P. 3D energetic metal-organic frameworks: Synthesis and properties of high energy materials. Angew. Chem. Int. Ed. 2013, 52, 14031-14035.

3. Li, Y.C.; Qi, C.; Li, S.H.; Zhang, H.J.; Sun, C.H.; Yu, Y.Z.; Pang, S.P. 1,1'-Azobis-1,2,3-triazole:

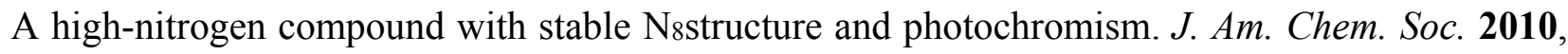
132, 12172-12173.

4. Tang, Y.X.; Yang, H.W.; Cheng, G.B. Synthesis and characterization of a stable, catenated $\mathrm{N}_{11}$ energetic salt. Angew. Chem. Int. Ed. 2013, 52, 4875-4877.

5. Jung, J.W.; Kim, K.J. Effect of supersaturation on the morphology of coated surface in coating by solution crystallization. Ind. Eng. Chem. Res. 2011, 50, 3475-3482.

6. Landenberger, K.B.; Matzger, A.J. Cocrystals of 1,3,5,7-tetranitro-1,3,5,7-tetrazacyclooctane (HMX). Cryst. Growth Des. 2012, 12, 3603-3609.

7. Yang, Z.W.; Li, H.Z.; Zhou, X.Q.; Zhang, C.Y. Characterization and properties of a novel energetic-energetic cocrystal explosive composed of HNIW and BTF. Cryst. Growth Des. 2012, 12, 5155-5158.

8. Landenberger, K.B.; Bolton, O.; Matzger, A.J. Two isostructural explosive cocrystals with significantly different thermodynamic stabilities. Angew. Chem. Int. Ed. 2013, 52, 6468-6471.

9. Landenberger, K.B.; Matzger, A.J. Cocrystal engineering of a prototype energetic material: Supramolecular chemistry of 2,4,6-trinitrotoluene. Cryst. Growth Des. 2012, 10, 5341-5347.

10. Bolton, O.; Matzger, A.J. Improved stability and smart-material functionality realized in an energetic cocrystal. Angew. Chem. Int. Ed. 2011, 50, 8960-8963.

11. Bolton, O.; Simke, L.R.; Matzger, A.J. High power explosive with good sensitivity: A 2:1 cocrystal of CL-20:HMX. Cryst. Growth Des. 2012, 12, 4311-4314. 
12. Zhang, H.B.; Guo, C.Y.; Wang, X.C.; Xu, J.J.; He, X.; Liu, Y.; Liu, X.F.; Huang, H.; Sun, J. Five energetic cocrystals of BTF by intermolecular hydrogen bond and $\pi$-stacking interactions. Cryst. Growth Des. 2013, 2, 679-687.

13. Dobrowolska, W.S.; Bator, G.; Sobczyk, L. The (2:1) complex of picric acid with tetramethylpyrazine: The structure, IR spectra and tunnel splitting of methyl groups. J. Mol. Struct. 2010, 975, 298-302.

14. Bertolasi, V.; Gilli, P.; Gilli, G. Hydrogen bonding and Electron Donor-Acceptor (EDA) interactions controlling the crystal packing of picric acid and its adducts with nitrogen bases. Their rationalization in terms of the $\mathrm{pKa}$ equalization and electron-pair saturation concepts. Cryst. Growth Des. 2011, 11, 2724-2735.

15. Liu, Y.; Wang, G.X.; Gong, X.D. 2,4-Diazido-5-iodo-pyrimidine crystal under high pressure: A comparison of DFT and DFT-D studies. Comput. Theor. Chem. 2012, 1000, 60-69.

16. Liu, Y.; Du, H.C.; Wang, G.X.; Gong, X.D. Comparative theoretical studies of high pressure effect on polymorph I of 2,2',4,4',6,6'-hexanitroazobenzene crystal. Struct. Chem. 2012, 23, 1631-1642.

17. Saminathan, K.; Sivakumar, K. A 2:1 complex of 2-nitro-aniline and picric acid. Acta Crystallogr. Sect. E Struct. Rep. Online 2007, 63, 354-356.

18. Thakuria, H.; Borah, B.M.; Pramanik, A.; Das, G. Solid state synthesis and hierarchical supramolecular self-assembly of organic salt cocrystals. J. Chem. Crystallogr. 2007, 37, 807-816.

19. Sheldrick, G.M. Foundations of crystallography. J. Acta Crystallogr. Sect. A Found. Crystallogr. 2008, 64, 112-122.

20. Janiak, C. A critical account on $\pi-\pi$ stacking in metal complexes with aromatic nitrogen-containing ligands. J. Chem. Soc. Dalton Trans. 2000, 21, 3885-3896.

21. Zhang, C.Y.; Xue, X.G.; Cao, Y.F.; Zhou, Y.; Li, H.Z.; Zhou, J.H.; Gao, T. Intermolecular friction symbol derived from crystal information. Cryst. Eng. Comm. 2013, 15, 6837-6844.

22. Spackman, M.A.; Jayatilaka, D. Hirshfeld surface analysis. Cryst. Eng. Comm. 2009, 11, 19-32.

23. Spackman, M.A.; McKinnon, J.J. Fingerprinting intermolecular interactions in molecular crystals. Cryst. Eng. Comm. 2012, 4, 378-392.

24. Lorenzo, I.; Graña, A.M. Stacking and hydrogen bond interactions between adenine and gallic acid. J. Mol. Model. 2013, 19, 5293-5299.

25. Zhang, C.Y. Review of the establishment of nitro group charge method and its applications. J. Hazard. Mater. 2009, 161, 21-28.

(C) 2015 by the authors; licensee MDPI, Basel, Switzerland. This article is an open access article distributed under the terms and conditions of the Creative Commons Attribution license (http://creativecommons.org/licenses/by/4.0/). 